ISSN 2079-9721

www.mdpi.com/journal/diseases/

Review

\title{
The Significance of Pulmonary Artery Size in Pulmonary Hypertension
}

Kamonpun Ussavarungsi ${ }^{1, *}$, Joseph P. Whitlock ${ }^{2}$, Taylor A. Lundy ${ }^{3}$, Ivan D. Carabenciov ${ }^{3}$, Charles D. Burger ${ }^{1}$ and Augustine S. Lee ${ }^{1}$

1 Pulmonary and Critical Care Medicine, Mayo Clinic, 4500 San Pablo Road, Jacksonville, FL 32224, USA; E-Mails: burger.charles@mayo.edu (C.D.B.); lee.augustine@mayo.edu (A.S.L.)

2 Diagnostic Radiology, Mayo Clinic, 4500 San Pablo Road, Jacksonville, FL 32224, USA; E-Mail: whitlock.joseph@mayo.edu

3 Clinical Research Internship Study Program, Mayo Clinic, San Pablo Road, Jacksonville, FL 32224, USA; E-Mails: taylor.lundy@unf.edu (T.A.L.); dcarabenciov@gmail.com (I.D.C.)

* Author to whom correspondence should be addressed; E-Mail: Ussavarungsi.kamonpun@mayo.edu; Tel.: +1-904-953-2861; Fax: +1-904-953-2082.

Received: 12 June 2014; in revised form: 21 July 2014 / Accepted: 25 July 2014 /

Published: 31 July 2014

\begin{abstract}
Pulmonary hypertension (PH) has been found to have significant morbidity and mortality. The treatment of $\mathrm{PH}$ has advanced considerably with increasingly more effective and safer options. With an increasing effort to diagnose patients early, non-invasive techniques are often used to screen those patients likely to have PH. Computerized tomography (CT) chest scans are increasingly utilized in the evaluation of patients with exertional dyspnea, including those with suspected $\mathrm{PH}$. The main role of the CT scan is to evaluate for any associated underlying diseases. There have been attempts to address the utility of CT to predict the presence of $\mathrm{PH}$. This article reviews previously published investigations to summarize the relationship between pulmonary artery dimensions and $\mathrm{PH}$ to determine both the strength of the correlation and its discriminatory ability for use in clinical practice.
\end{abstract}

Keywords: pulmonary hypertension; pulmonary artery diameter; computed tomography; lung; diagnosis; screening 


\section{Introduction}

Although there have been significant advances in the treatment of pulmonary hypertension ( $\mathrm{PH})$, there remains significant morbidity and mortality [1-3]. With increasingly more effective and safer pharmacological therapy for pulmonary arterial hypertension (PAH), outcomes may be improved by earlier detection of $\mathrm{PH}$ [3]. Screening algorithms have been proposed to facilitate the timely and accurate diagnosis of $\mathrm{PH}$, utilizing a combination of echocardiographic, physiologic (lung function), and radiologic non-invasive techniques [4,5], before proceeding to a definitive right heart catheterization (RHC) for confirmation.

Computed tomography (CT) chest scans have largely supplanted chest x-rays in patients with $\mathrm{PH}$, partly due to its ability to detect thromboembolism in some cases, but also to identify any diffuse parenchymal lung diseases that may not be evident in $15 \%$ of chest $\mathrm{x}$-rays [6,7]. With advances in CT technology and its wide availability, there have been attempts to address the utility of CT to predict the presence of $\mathrm{PH}$.

The pulmonary artery (PA) is a more compliant vessel than the systemic arterial system, and is thus more sensitive to changes in pressure and volume. As a result, an increase in mean pulmonary arterial pressure (MPAP) should correlate with pulmonary artery diameter. A variety of PA dimensions have been explored to see if there is any association with both the presence and severity of $\mathrm{PH}$, including the PA diameter, the cross-sectional area, the ratio of the diameter to the bronchus, the ratio of the diameter to the pulmonary vein, the ratio of diameter to the aortic diameter, and multiple regression methods assessing dimension of the main and branching pulmonary arteries [8-32]. Based on such observations, we have found radiologists formally reporting on the PA size and suggesting the presence or absence of $\mathrm{PH}$, which often has led to changes in clinical behavior without a clear justification otherwise.

In this review, we have compiled, for the first time, all published investigations exploring the relationship between PA dimensions and $\mathrm{PH}$ to determine both the strength of the correlation and whether it has adequate discriminatory ability for use in clinical practice. Extrapolating from our own series, we hypothesized that, although the PA size should correlate with PA pressures, its discriminatory ability is poor and over utilized clinically.

\section{Methods}

An OVID Medline literature search was used to identify all English and human studies relating PA size as measured on a CT of the chest in patients with PH confirmed by RHC (or in some series as suggested on echocardiography) between 1991 and 2014. PA dimensions are expressed in millimeters $(\mathrm{mm})$ with their standard deviation $( \pm \mathrm{SD})$. In the brief report of our series, consecutive patients referred to the PH clinic were identified between 2006 and 2010. CT measures were blinded to clinical history and were done by three separate reviewers.

\section{Pathophysiology}

The pulmonary circulation is a high flow, low pressure system. It has a lower resistance and is more compliant than the systemic circulation, owing to the abundance of vasculature in parallel and a lower 
transpulmonary pressure gradient. The factors that determine the size of a vessel depend on several physiologic variables and any underlying pathology. Fundamentally, it is the volume of blood within the PA that relates to the size of the PA as measured on the CT, with the indirect factors of pressure and vessel compliance contributing. From this, it is easy to understand how PA size will correlate with PA pressures, but with an often incorrect assumption of constant compliance and blood volume. Practically, we can measure the MPAP, cardiac output, PA occlusion pressure (as a surrogate for left ventricular end-diastolic pressure) from RHC and derive secondarily, the pulmonary vascular resistance [19,20]. Additionally, there are variations due to anthropomorphic factors that can be considered such as age, gender, height, and body surface area (BSA) [20,33]. Pathologic factors within the vessel itself may include atherosclerosis, endothelial proliferation, and occlusion from thrombi [20]. In idiopathic PAH (i.e., diagnostic group 1.1 pulmonary arterial hypertension), the peripheral pulmonary vessels are characterized by the degeneration of the elastic lamina with replacement by fibrous tissue, along with intimal proliferation and hypertrophy of the muscular layer in the media of the arteries [34-36]. This downstream occlusion with subsequent increase in resistance is what should lead to proximal increase in the main PA pressure and size. The conditions that cause mechanical changes to the mediastinal vasculature by compression, traction, or shifting, as well as pathologic changes in the heart or lungs, such as from congenital heart disease, prior surgeries, or radiation, can cause additional anatomic distortion. Despite limited investigations that address this [20,37-39], we suspect that technical issues in the imaging of the PA (e.g., body position, different acquisition protocols and reconstruction algorithms, depth of inspiration, the use of contrast, and reduced intraand inter-rater reliability) may also affect the accurate measure of the PA size and further confound the relationship between the PA size and PA pressures. Although logical deduction would indicate that the PA size should correlate nicely with $\mathrm{PH}$ and $\mathrm{PH}$ severity, there are a multitude of factors that may potentially make the measurement unreliable or inaccurate. Lastly, the reader should appreciate that "PH" is simply a hemodynamic condition defined by a MPAP $\geq 25 \mathrm{mmHg}$ at rest as documented by $\mathrm{RHC}$; therefore, is more a pathophysiologic state than a specific disease or diagnosis.

\section{What Is the Normal Reference Value?}

Many studies have tried to determine the normal range of the main PA size. Typically, the transverse axial diameter of the main PA at the level of its bifurcation is measured. This landmark is easy to define anatomically and is highly reproducible [20], and at this same level, the ascending aorta can also be measured to calculate the ratio of main PA to the aortic diameter (PA/Ao) (Figure 1), as a means of "normalizing" for differences in anthropomorphic factors.

Several published studies provide normative data. Edwards et al. reported that the mean PA size, as measured on a non-contrast CT, was $27.2 \mathrm{~mm}$ (SD 0.6) in 100 study participants with no history of cardiopulmonary disease; however, no RHC was performed [15]. Karazincir et al. reported a mean diameter of the main PA of $26.6 \mathrm{~mm}$ (SD 2.9) in a cohort of 112 patients who had no evidence of pulmonary disease and documented normal PA pressures by echocardiogram (MPAP $\leq 25 \mathrm{mmHg}$ ) [40]. The Karazincir study was performed as clinically indicated rather than by research protocol; therefore, the mean PA diameter may be falsely elevated. Kuriyama et al. reported a somewhat smaller mean PA size of $24.2 \mathrm{~mm}$ (SD 2.2) [17]. The possible discrepancy may be due to the differences in CT 
techniques and race. The majority of Kuriyama et al.'s study participants were of Japanese origin, while Edward et al.'s participants were Caucasian. It is important to note that overlapping hemodynamic definitions of $\mathrm{PH}$ in the various studies may confound the results. Indeed, the Karizincir study uses a definition that actually includes PH, i.e., a MPAP of $25 \mathrm{mmHg}$. In addition, a normal MPAP is less than $20 \mathrm{mmHg}$ as suggested by RHC data from 1,184 healthy individuals that reported a normal MPAP of $14 \mathrm{mmHg}$ (SD 3.3) [41].

Figure 1. Measurements of main pulmonary artery and ascending aorta at the level of bifurcation. The main pulmonary artery (PA) size is typically taken at the level of the bifurcation of the main pulmonary artery perpendicular to the vessel wall. The aortic dimension of the ascending aorta is taken at the same level to calculate the PA to the aortic diameter $(\mathrm{PA} / \mathrm{Ao})$ ratio. The diameter is determined using the internal diameter in the contrast-enhanced image.

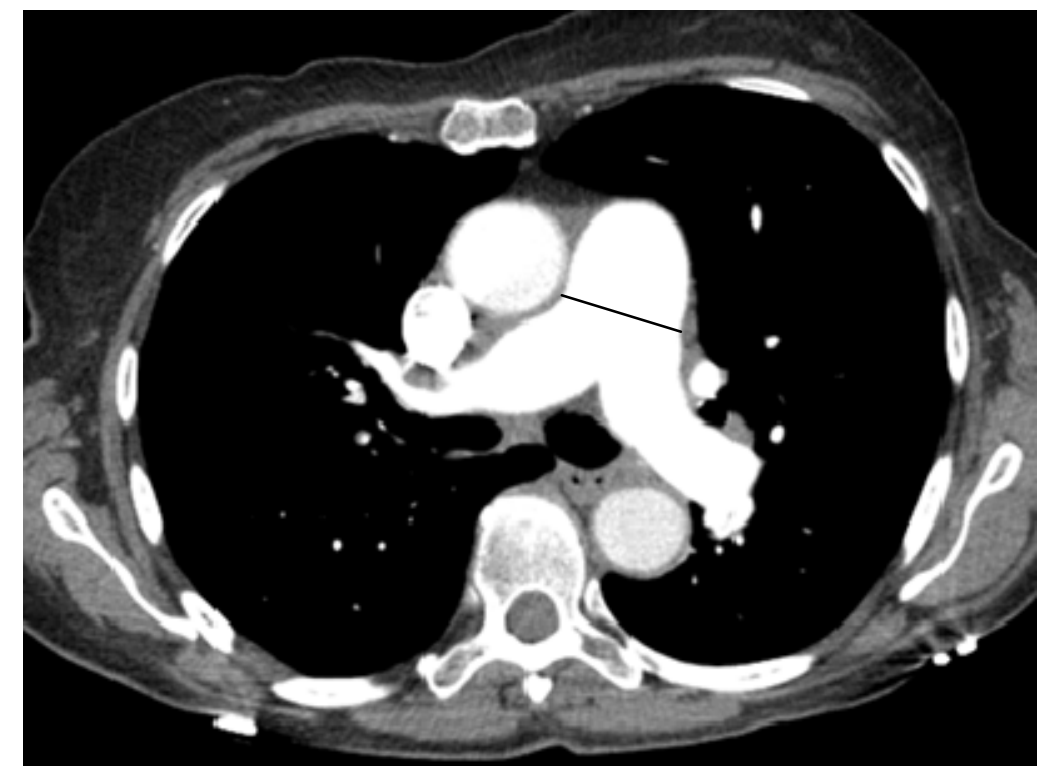

There are also reported gender differences, with men having a slightly higher average PA size of $27.0 \mathrm{~mm}$ (SD 2.8) versus $25.9 \mathrm{~mm}$ (SD 3.0) in women $(\mathrm{P}=0.048)$. However, it appears the gender differences could largely be accounted for by differences in the BSA between men and women [20,42] for the main PA size, with the exception of the right PA. Similarly, gender differences in the size of the aorta could also be explained by adjusting for the BSA, but notably not the ascending aorta [42].

The largest population cohort in which PA dimensions were assessed by CT comes from the Framingham Heart Study of 3171 participants (mean age 51 years, 51\% men), of whom 706 were identified as asymptomatic without any cardiopulmonary risk factors. The main PA size in this reference subset was $24.7 \mathrm{~mm}$ (SD 2.7) and the PA/Ao was 0.80 (SD 0.09) [33]. Interestingly, there was a weak inverse correlation between age and the main PA size in men $(\mathrm{r}=-0.11, \mathrm{p}=0.04)$, although for the entire cohort, the correlation was direct $(r=0.10, p<0.0001)$. Height also weakly correlated with the main PA size (men: $\mathrm{r}=0.18, \mathrm{P}<0.0001$; women $\mathrm{r}=0.24, \mathrm{p}<0.0001$ ), but was stronger again for the BSA (men: $r=0.41, p<0.0001$; women: $r=0.42 ; p<0.0001$ ). Using the subset of asymptomatic study participants without cardiopulmonary risk factors, they established a 90th percentile gender-specific cutoff value for main PA of $29 \mathrm{~mm}$ for men and $27 \mathrm{~mm}$ for women [33]. 


\section{Correlation between PA Size and PH}

Numerous studies have investigated the correlation between CT measurements of the PA and the presence and severity of PH (Tables 1 and 2). Overall, the measurement of the main PA size by using CT shows a moderate to strong correlation with $\mathrm{PH}(\mathrm{r} \sim 0.4-0.7)$.

Table 1. Studies of CT measurements in patients with PH.

\begin{tabular}{|c|c|c|c|}
\hline Studies & Patients & Measurement & $\begin{array}{l}\text { Correlation or Operating } \\
\text { Characteristics }\end{array}$ \\
\hline \multicolumn{4}{|c|}{ Predominantly PH WHO group 1} \\
\hline $\begin{array}{l}\text { Edwards PD et al. } \\
1998[15]\end{array}$ & $\begin{array}{l}100 \text { normal subjects } \\
12 \text { patients with PAH }\end{array}$ & $\begin{array}{l}\text { Main PA in predicting PH } \\
\text { (MPAP }>20 \mathrm{mmHg} \text { ), } \\
\text { cut-off } 33.2 \mathrm{~mm}\end{array}$ & sensitivity $58 \%$, specificity $95 \%$ \\
\hline $\begin{array}{l}\text { Grubstein A et al. } \\
2008[26]\end{array}$ & $\begin{array}{l}38 \text { patients with } \mathrm{PH} \\
\text { (primary } \mathrm{PH}, \mathrm{n}=20 \text { ) } \\
22 \text { control }\end{array}$ & $\begin{array}{l}\text { Main PA vs. PASP by echo } \\
\text { Main PA vs. RHC }\end{array}$ & $\begin{array}{l}r=0.43 \\
r=0.38\end{array}$ \\
\hline $\begin{array}{l}\text { Rajaram S et al. } \\
2012[30]\end{array}$ & $\begin{array}{l}81 \text { patients with } \\
\text { connective tissue } \\
\text { disease }\end{array}$ & $\begin{array}{l}\text { Main PA } v s . \text { MPAP } \\
\text { PA/Ao ratio } v s . \text { MPAP } \\
\text { Main PA in predicting PH } \\
\text { (MPAP } \geq 25 \mathrm{mmHg} \text { ), } \\
\text { cut-off } 29 \mathrm{~mm} \\
\text { PA/Ao ratio }>1 \text { in predicting }\end{array}$ & $\begin{array}{l}r=0.37 \\
r=0.43 \\
\text { sensitivity } 59 \% \text {, specificity } 73 \% \text {, } \\
\text { AUC }=0.71 \\
\text { sensitivity } 54 \% \text {, specificity } 74 \% \text {, } \\
\text { AUC }=0.73\end{array}$ \\
\hline \multicolumn{4}{|c|}{ Predominantly PH WHO group 2} \\
\hline $\begin{array}{l}\text { Kuriyama et al. } \\
1984 \text { [17] }\end{array}$ & $\begin{array}{l}32 \text { patients with } \\
\text { cardiopulmonary } \\
\text { diseases } \\
\text { (Most of the patients } \\
\text { had cardiac disease, } \\
\mathrm{n}=25 \text { ) } \\
26 \text { control }\end{array}$ & $\begin{array}{l}\text { Main PA vs. MPAP } \\
\text { Main PA in predicting PH } \\
\text { (MPAP }>18 \mathrm{mmHg} \text { ), } \\
\text { cut-off } 28.6 \mathrm{~mm}\end{array}$ & $\begin{array}{l}r=0.83 \\
\text { sensitivity } 69 \% \text {, specificity } 100 \%\end{array}$ \\
\hline $\begin{array}{l}\text { Burger IA et al. } \\
2011[10]\end{array}$ & $\begin{array}{l}100 \text { patients } \\
\text {-CAD } \\
\text { assessment }(60) \\
\text {-Dyspnea }(40) \\
\text { with PH }(\mathrm{n}=37)\end{array}$ & $\begin{array}{l}\text { Main PA in predicting } \mathrm{PH} \\
\text { (Echo } \mathrm{RV} / \mathrm{RA} \text { gradient } \\
\geq 30 \mathrm{mmHg} \text { ), cut-off } 30 \mathrm{~mm}\end{array}$ & $\begin{array}{l}\text { sensitivity } 78 \% \text {, specificity } 91 \% \text {, } \\
\text { PPV } 83 \%, A U C=0.85\end{array}$ \\
\hline
\end{tabular}


Table 1. Cont.

\begin{tabular}{|c|c|c|c|}
\hline Studies & Patients & Measurement & $\begin{array}{l}\text { Correlation or Operating } \\
\text { Characteristics }\end{array}$ \\
\hline \multicolumn{4}{|c|}{ Predominantly PH WHO group 2} \\
\hline $\begin{array}{l}\text { Chan AL et al. } \\
2011 \text { [9] }\end{array}$ & $\begin{array}{l}101 \text { hospitalized } \\
\text { patients } \\
\text { (Most of the patients } \\
\text { had cardiac disease, } \\
\mathrm{n}=70 \text { ) }\end{array}$ & $\begin{array}{l}\text { Main PA in predicting PH } \\
\text { (MPAP } \geq 25 \mathrm{mmHg} \text { ), } \\
\text { cut-off } 29 \mathrm{~mm} \\
\text { PA/Ao ratio }>0.84 \text { in } \\
\text { predicting } \mathrm{PH}\end{array}$ & $\begin{array}{l}\text { sensitivity } 67.9 \% \text {, specificity } \\
56.3 \% \text {, AUC }=0.68 \\
\text { sensitivity } 79.2 \% \text {, specificity } \\
50 \%, \text { AUC }=0.68\end{array}$ \\
\hline $\begin{array}{l}\text { Kam JC et al. } \\
2013[16]\end{array}$ & $\begin{array}{l}40 \text { patients with left- } \\
\text { sided cardiac disease }\end{array}$ & $\begin{array}{l}\text { Main PA in predicting PH } \\
\text { (MPAP } \geq 25 \mathrm{mmHg} \text {, } \\
\text { cut-off } 33.3 \mathrm{~mm} \\
\text { Cut-off }<27.3\end{array}$ & $\begin{array}{l}\text { specificity } 100 \% \text {, PPV } 100 \% \text {, } \\
\text { AUC }=0.95 \\
\text { sensitivity } 100 \% \text {, NPV } 100 \%\end{array}$ \\
\hline \multicolumn{4}{|c|}{ Predominantly PH WHO group 3} \\
\hline $\begin{array}{l}\text { Haimovici et al. } \\
1997 \text { [25] }\end{array}$ & $\begin{array}{l}55 \text { patients, } \\
\text { candidates for heart- } \\
\text { lung transplantation } \\
\text {-Chronic lung disease } \\
\text { e.g., COPD, IPF (45) } \\
\text {-PVD (10) }\end{array}$ & $\begin{array}{l}\text { Main PA vs. MPAP } \\
\text { Main PA/BSA vs. MPAP } \\
\text { Left main PA vs. MPAP } \\
\text { Left main PA/BSA vs. MPAP } \\
\text { Main and left main PA/BSA } \\
v s . \text { MPAP }\end{array}$ & $\begin{array}{l}r=0.67 \\
r=0.66 \\
r=0.69 \\
r=0.71 \\
r=0.87\end{array}$ \\
\hline $\begin{array}{l}\text { Ng CS et al. } \\
1999[20]\end{array}$ & $\begin{array}{l}50 \text { patients with } \\
\text { cardiopulmonary } \\
\text { diseases } \\
\text { (Most of the patients } \\
\text { had chronic lung } \\
\text { disease, } \mathrm{n}=33 \text { ) }\end{array}$ & $\begin{array}{l}\text { Main PA vs. MPAP } \\
\text { PA/Ao ratio } v s . \text { MPAP } \\
\text { Main PA diameter cut-off of } \\
30 \mathrm{~mm} \text { in predicting PH } \\
\text { (MPAP }>20 \mathrm{mmHg} \text { ) } \\
\text { PA/Ao }>1 \text { in predicting } \mathrm{PH} \\
(\mathrm{MPAP}>20 \mathrm{mmHg} \text { ) }\end{array}$ & $\begin{array}{l}r=0.74 \\
r=0.74 \\
\text { sensitivity } 68 \% \text {, specificity } 100 \% \text {, } \\
\text { PPV } 100 \% \\
\text { sensitivity } 70 \% \text {, specificity } 92 \% \text {, } \\
\text { PPV } 96 \%\end{array}$ \\
\hline $\begin{array}{l}\text { Tan RT et al. } \\
1998 \text { [23] }\end{array}$ & $\begin{array}{l}36 \text { patients with } \mathrm{PH} \\
9 \text { control } \\
\text {-Chronic lung } \\
\text { disease, e.g., COPD, } \\
\text { ILD (24) } \\
\text {-PVD (12) }\end{array}$ & $\begin{array}{l}\text { Main PA vs. MPAP } \\
\text { Main PA in predicting PH } \\
\text { (MPAP }>20 \mathrm{mmHg} \text { ), } \\
\text { cut-off } 29 \mathrm{~mm}\end{array}$ & $\begin{array}{l}r=0.12(\mathrm{NS}) \\
\text { sensitivity } 87 \% \text {, specificity } 89 \% \text {, } \\
\text { PPV } 97 \% \text { and sensitivity } 84 \% \text {, } \\
\text { specificity } 75 \%, \text { PPV } 95 \% \text { in } \\
\text { subgroup of parenchymal lung } \\
\text { disease }(n=28)\end{array}$ \\
\hline
\end{tabular}


Table 1. Cont.

\begin{tabular}{|c|c|c|c|}
\hline Studies & Patients & Measurement & $\begin{array}{l}\text { Correlation or Operating } \\
\text { Characteristics }\end{array}$ \\
\hline \multicolumn{4}{|c|}{ Predominantly PH WHO group 3} \\
\hline $\begin{array}{l}\text { Iyer AS et al. } \\
2014 \text { [29] }\end{array}$ & $\begin{array}{l}60 \text { patients with } \\
\text { COPD, referred for } \\
\text { transplantation }\end{array}$ & $\begin{array}{l}\text { Main PA vs. MPAP } \\
\text { PA/Ao ratio vs. MPAP } \\
\text { PA/Ao ratio vs. MPAP } \\
\text { adjusting for age, race, sex, } \\
\text { BMI, resting oxygen } \\
\text { saturation, sleep apnea, } \\
\text { congestive heart failure, and } \\
\text { diabetes mellitus } \\
\text { PA/Ao }>1 \text { in predicting } \mathrm{PH} \\
(\mathrm{MPAP}>25 \mathrm{mmHg})\end{array}$ & $\begin{array}{l}r=0.60 \\
r=0.56 \\
r=0.30 \\
\text { sensitivity } 73 \%, \text { specificity } 84 \% \text {, } \\
\text { AUC }=0.83\end{array}$ \\
\hline \multicolumn{4}{|c|}{ Predominantly PH WHO group 4} \\
\hline $\begin{array}{l}\text { Moore et al. } \\
1988 \text { [19] }\end{array}$ & $\begin{array}{l}24 \text { patients with } \\
\text { primary } \mathrm{PH} \text { and } \\
\text { CTEPH }\end{array}$ & Main PA vs. MPAP & No correlation, $r$ was not reported \\
\hline $\begin{array}{l}\text { Schmidt et al. } \\
1996 \text { [22] }\end{array}$ & $\begin{array}{l}50 \text { patients with } \\
\text { CTEPH }\end{array}$ & Main PA vs. MPAP & $r=0.43$ \\
\hline $\begin{array}{l}\text { Sanal S et al. } \\
2006[21]\end{array}$ & $\begin{array}{l}190 \text { patients with } \\
\text { acute pulmonary } \\
\text { embolism }\end{array}$ & $\begin{array}{l}\text { Main PA in predicting } \mathrm{PH} \\
\text { (Echo PASP } \geq 50 \mathrm{mmHg} \text { ), } \\
\text { cut-off } 28.6 \mathrm{~mm} \\
\text { PA/Ao ratio }>1 \text { in } \\
\text { predicting } \mathrm{PH}\end{array}$ & $\begin{array}{l}\text { sensitivity } 75 \% \text {, specificity } 75 \% \text {, } \\
\text { PPV } 52 \% \\
\text { sensitivity } 59 \% \text {, specificity } 82 \% \text {, } \\
\text { PPV } 55 \%\end{array}$ \\
\hline \multicolumn{4}{|c|}{ Mixed PH groups } \\
\hline $\begin{array}{l}\text { Abel E et al. } \\
2012 \text { [11] }\end{array}$ & $\begin{array}{l}27 \text { patients with } \mathrm{PH} \\
\text { defined as } \\
\mathrm{MPAP} \geq 25 \mathrm{mmHg}\end{array}$ & $\begin{array}{l}\text { Main PA vs. MPAP } \\
\text { PA/Ao ratio vs. MPAP }\end{array}$ & $\begin{array}{l}r=0.53 \\
r=0.41\end{array}$ \\
\hline $\begin{array}{l}\text { Dornia C et al. } \\
2012 \text { [28] }\end{array}$ & $\begin{array}{l}114 \text { patients with } \mathrm{PH} \\
\text { defined as } \\
\mathrm{MPAP} \geq 25 \mathrm{mmHg} \\
58 \text { control with } \\
\mathrm{MPAP}<20 \mathrm{mmHg}\end{array}$ & $\begin{array}{l}\text { Main PA in predicting } \mathrm{PH} \\
\text { (MPAP } \geq 25 \mathrm{mmHg} \text { ), } \\
\text { cut-off } 29 \mathrm{~mm} \\
\text { PA/Ao ratio }>1 \text { in } \\
\text { predicting } \mathrm{PH}\end{array}$ & $\begin{array}{l}\text { sensitivity } 93.9 \% \text {, specificity } \\
62.1 \% \text {, PPV } 82.9 \% \text {, NPV } 83.7 \% \text {, } \\
\text { AUC }=0.93 \\
\text { sensitivity } 63.2 \% \text {, specificity } \\
93.1 \% \text {, PPV } 94.7 \% \text {, NPV } 56.3 \% \text {, } \\
\text { AUC }=0.88\end{array}$ \\
\hline
\end{tabular}


Table 1. Cont.

\begin{tabular}{|c|c|c|c|}
\hline Studies & Patients & Measurement & $\begin{array}{l}\text { Correlation or Operating } \\
\text { Characteristics }\end{array}$ \\
\hline \multicolumn{4}{|l|}{ Mixed PH groups } \\
\hline $\begin{array}{l}\text { Mahammdi A et al. } \\
2013 \text { [18] }\end{array}$ & $\begin{array}{l}298 \text { patients with } \mathrm{PH} \\
\text { defined as } \\
\text { MPAP } \geq 25 \mathrm{mmHg} \\
102 \text { control with } \\
\text { MPAP }<25 \mathrm{mmHg}\end{array}$ & $\begin{array}{l}\text { Main PA vs. MPAP } \\
\text { Main PA/BSA vs. MPAP } \\
\text { PA/Ao ratio vs. MPAP } \\
\text { Main PA in predicting PH } \\
\text { (MPAP } \geq 25 \mathrm{mmHg} \text { ), } \\
\text { cut-off } 31.5 \mathrm{~mm} \\
\text { PA/Ao ratio }>1 \text { in } \\
\text { predicting PH } \\
\text { Combined Main PA > } 29.5 \\
\text { and PA/Ao ratio }>1\end{array}$ & $\begin{array}{l}r=0.51 \\
r=0.35 \\
r=0.54 \\
\text { sensitivity } 52 \% \text {, specificity } 90 \% \\
\text { sensitivity } 71 \% \text {, specificity } 76 \% \\
\text { AUC }=0.80\end{array}$ \\
\hline $\begin{array}{l}\text { Lange } \mathrm{T} \text { et al. } \\
2013[31]\end{array}$ & $\begin{array}{l}78 \text { patients } \\
\mathrm{n}=52, \mathrm{MPAP} \leq \\
20 \mathrm{mmHg} \\
\mathrm{n}=26, \mathrm{MPAP} \\
21-24 \mathrm{mmHg}\end{array}$ & $\begin{array}{l}\text { Main PA } v s . \text { MPAP } \\
\text { Main PA in predicting } \\
\text { borderline PH, } \\
\text { cut-off } 29 \mathrm{~mm}\end{array}$ & $\begin{array}{l}r=0.49 \\
\text { sensitivity } 77 \% \text {, specificity } 62 \% \text {, } \\
\text { PPV } 50 \% \text {, NPV } 84 \%, \\
\text { AUC }=0.73\end{array}$ \\
\hline $\begin{array}{l}\text { Corson N et al. } \\
2014 \text { [13] }\end{array}$ & $\begin{array}{l}175 \text { patients with } \mathrm{PH} \\
16 \text { patients without } \\
\text { PH (by RHC) } \\
114 \text { normal (but no } \\
\text { RHC) }\end{array}$ & $\begin{array}{l}\text { Main PA vs. MPAP } \\
\text { Main PA in predicting PH } \\
\text { (MPAP } \geq 25 \mathrm{mmHg} \text { ), } \\
\text { cut-off } 29 \mathrm{~mm} \\
\text { PA/Ao ratio }>1 \text { in predicting } \\
\text { PH }\end{array}$ & $\begin{array}{l}r=0.34 \\
\text { sensitivity } 89 \% \text {, specificity } 83 \% \text {, } \\
\text { AUC }=0.96 \\
\text { sensitivity } 89 \% \text {, specificity } 82 \% \text {, } \\
\text { AUC }=0.94\end{array}$ \\
\hline
\end{tabular}

AUC: area under curve; BSA: body surface area; CAD: coronary artery disease; COPD: chronic obstructive pulmonary disease; CT: computed tomography; CTEPH: chronic thromboembolic pulmonary hypertension; ILD: interstitial lung disease; IPF: idiopathic pulmonary fibrosis; MPAP: mean pulmonary artery pressure; NPV: negative predictive value; PA: pulmonary artery; PAH: pulmonary arterial hypertension; PASP: pulmonary arterial systolic pressure; PA/Ao ratio: ratio of the diameter of the pulmonary artery to the diameter of the aorta; PH: pulmonary hypertension; PPV: positive predictive value; PVD: peripheral vascular disease; RHC: right heart catheterization; RV/RA: right ventricular/right atrial; WHO: World Health Organization. 
Table 2. CT measurement studies in patients with parenchymal lung disease (Subset of WHO Group 3 with fibrotic lung disease).

\begin{tabular}{|c|c|c|c|}
\hline Studies & Patients & Measurement & $\begin{array}{l}\text { Correlation or Operating } \\
\text { Characteristics }\end{array}$ \\
\hline $\begin{array}{l}\text { Zisman D et al. } \\
2007[24]\end{array}$ & $\begin{array}{l}65 \text { patients with } \\
\text { idiopathic pulmonary } \\
\text { fibrosis }\end{array}$ & $\begin{array}{l}\text { Main PA vs. MPAP } \\
\text { Main PA/BSA vs. MPAP } \\
\text { PA/Ao ratio } v s . \text { MPAP }\end{array}$ & $\begin{array}{l}r=0.14(\mathrm{NS}) \\
r=0.13(\mathrm{NS}) \\
r=0.20(\mathrm{NS})\end{array}$ \\
\hline $\begin{array}{l}\text { Devaraj A et al. } \\
2008[27]\end{array}$ & $\begin{array}{l}77 \text { patients } \\
\text { With (group A, } \\
\mathrm{n}=30 \text { ) } \\
\text { Without (group B, } \\
\mathrm{n}=47 \text { ) } \\
\text { fibrotic lung disease }\end{array}$ & $\begin{array}{l}\text { Group A } \\
\text { Main PA vs. MPAP } \\
\text { PA/Ao ratio vs. MPAP } \\
\text { Group B } \\
\text { Main PA vs. MPAP } \\
\text { PA/Ao ratio vs. MPAP }\end{array}$ & $\begin{array}{l}r=0.23(\mathrm{NS}) \\
r=0.54 \\
r=0.67 \\
r=0.72\end{array}$ \\
\hline $\begin{array}{l}\text { Alhamad EH et al. } \\
2011 \text { [12] }\end{array}$ & $\begin{array}{l}134 \text { patients } \\
\text { With ILD (group A, } \\
\mathrm{n}=100 \text { ) } \\
\text { Without ILD } \\
\text { (group } \mathrm{B}, \mathrm{n}=34 \text { ) }\end{array}$ & $\begin{array}{l}\text { Group A } \\
\text { Main PA vs MPAP } \\
\text { PA/Ao ratio } v s . \text { MPAP } \\
\text { Group B } \\
\text { Main PA vs. MPAP } \\
\text { PA/Ao ratio } v s . \text { MPAP } \\
\text { Group A } \\
\text { Main PA in predicting PH } \\
\text { (MPAP } \geq 25 \mathrm{mmHg} \text { ), } \\
\text { cut-off } 25 \mathrm{~mm} \\
\text { Group B } \\
\text { Main PA in predicting PH } \\
\text { (MPAP } \geq 25 \mathrm{mmHg} \text { ), } \\
\text { cut-off } 31.6 \mathrm{~mm}\end{array}$ & $\begin{array}{l}r=0.30 \\
r=0.43 \\
r=0.70 \\
r=0.62 \\
\text { sensitivity } 86 \%, \text { specificity } \\
41 \%, \text { AUC }=0.65 \\
\text { sensitivity } 47 \%, \text { specificity } \\
93 \%, \text { AUC }=0.73\end{array}$ \\
\hline $\begin{array}{l}\text { Condliffe et al. } \\
2011[43]\end{array}$ & $\begin{array}{l}89 \text { scleroderma } \\
\text { CT within } 3 \text { months } \\
\text { of RHC, } n=81 \\
\text { Subset of patients } \\
\text { without ILD, } n=63\end{array}$ & $\begin{array}{l}\text { Main PA } v s \text { MPAP } \\
\text { PA/Ao ratio } v s . \text { MPAP } \\
\text { No ILD, } \mathrm{n}=63 \\
\text { Main PA vs. MPAP } \\
\text { PA/Ao ratio } v s . \text { MPAP }\end{array}$ & $\begin{array}{l}r=0.34 \\
r=0.42\end{array}$ \\
\hline
\end{tabular}


Table 2. Cont.

\begin{tabular}{|c|c|c|c|}
\hline Studies & Patients & Measurement & $\begin{array}{l}\text { Correlation or Operating } \\
\text { Characteristics }\end{array}$ \\
\hline $\begin{array}{l}\text { McCall RK et al. } \\
2014 \text { [32] }\end{array}$ & $\begin{array}{l}48 \text { scleroderma } \\
\text { Without ILD } \\
\text { (group A, } n=20 \text { ) } \\
\text { With ILD (group B, } \\
n=28 \text { ) } \\
\text { FVC }>70 \% \text { (group C, } \\
n=18 \text { ) } \\
\text { FVC }<70 \% \text { (group D, } \\
n=16 \text { ) }\end{array}$ & $\begin{array}{l}\text { Group A } \\
\text { Main PA } v s . \text { MPAP } \\
\text { PA/Ao ratio } v s . \text { MPAP } \\
\text { Group B } \\
\text { Main PA } v s . \text { MPAP } \\
\text { PA/Ao ratio } v s . \text { MPAP } \\
\text { Group C } \\
\text { Main PA } v s \text { MPAP } \\
\text { PA/Ao ratio } v s . \text { MPAP } \\
\text { Group D } \\
\text { Main PA } v s . \text { MPAP } \\
\text { PA/Ao ratio } v s . \text { MPAP } \\
\text { Main PA in predicting PH } \\
\text { (MPAP } \geq 25 \text { mmHg), } \\
\text { cut-off } 30.8 \text { mm }\end{array}$ & $\begin{array}{l}r=0.68 \\
r=0.50 \\
r=0.70 \\
r=0.47 \\
r=0.69 \\
r=0.66 \\
r=0.42(N S) \\
r=-0.09(N S) \\
\text { sensitivity } 81 \%, \text { specificity } \\
87 \%, \text { AUC }=0.86\end{array}$ \\
\hline
\end{tabular}

AUC: area under curve; BSA: body surface area; CT: computed tomography; FVC: forced vital capacity; ILD: interstitial lung disease; MPAP: mean pulmonary artery pressure; PA: pulmonary artery; PA/Ao ratio: ratio of the diameter of the pulmonary artery to the diameter of the aorta; PH: pulmonary hypertension; RHC: right heart catheterization.

However, Moore et al. reported no correlation between main PA and MPAP in 24 patients with history of primary $\mathrm{PH}$ and chronic thromboembolic pulmonary hypertension (CTEPH) [19]. The study observed that increasing main PA diameter was associated with decreased cardiac output $(\mathrm{r}=-0.75$, $\mathrm{p}<0.001)$ and increased pulmonary vascular resistance $(\mathrm{r}=0.61, \mathrm{p}<0.0005)$ in the patients with pulmonary vascular disease, explained by the obliteration of peripheral arteries [19].

The presence of significant parenchymal lung disease, which can distort the great vessel anatomy, also appears to affect the correlation between PH and PA size. Tan et al. [23] evaluated 36 patients with confirmed PH by RHC (defined by MPAP of at least $20 \mathrm{mmHg}$ ), but found no correlation between the main PA size and the MPAP. The lack of correlation was attributed to the presence of parenchymal lung disease and architectural distortion, which was present in the majority (24/36). Similarly, Zisman et al. and Devaraj et al. [24,27] also reported no significant correlation between the main PA size and MPAP in patients with fibrotic lung disease. In contrast, Alhamad et al. did find a moderate correlation between main PA size and MPAP in a larger series of patients with interstitial lung disease (ILD) [12] (Table 2). 
In systemic sclerosis and other connective tissues diseases, $\mathrm{PH}$ can be another manifestation apart from an ILD. A weak correlation between the main PA diameter and MPAP in connective tissue disease can be found [30], but appears to be mostly unaffected by the presence of ILD. For example, when Condliffe et al. evaluated patients with limited systemic sclerosis, a weak correlation was found $(\mathrm{r}=0.345, \mathrm{p}=0.002)$ between the main PA diameter and the MPAP, regardless of the extent of ILD [43]. In another cohort of 48 patients with scleroderma and ILD, the presence of mild to moderate fibrotic lung disease did not influence the correlation between the PA diameter and MPAP [32]. Notably, the correlation was reduced after adjusting for the BSA or the aortic diameter, and there was no correlation in the subset with more advanced restriction (FVC less than 70\%) [32].

Similarly, in a retrospective study of 65 patients with advance idiopathic pulmonary fibrosis, there was no significant correlation between the main PA diameter and MPAP [24], similar to observations by others [25]. These studies suggest that perhaps, in the context of fibrotic lung disease, the PA dimension is even less of a reliable parameter as an indicator for $\mathrm{PH}$. The mechanism for this is unclear, though a traction effect on the pulmonary vessels has been suggested [24].

\section{Diagnostic Utility of PA Size}

So given that there is evidence of some correlation between the PA size and PH severity, investigators have reported on whether specific thresholds could be used to facilitate the diagnosis or exclusion of $\mathrm{PH}$, and in particular, whether it might help with regards to the need for a confirmatory RHC. Depending on the study population, the cut-off selected for the PA diameter (25-33.3 mm), and the gold standard used, the reported sensitivity can range between $47 \%-100 \%$ and the specificity between $41 \%-100 \%[10,12,13,16-18,20-22,28]$. As anticipated, there is considerable overlap in the cut-off value and some overlap with what has been reported in the population without $\mathrm{PH}$ (Table 1). Although using the PA/Ao ratio has the potential to improve the diagnostic accuracy, its discriminatory ability appears also highly variable with a sensitivity of $59 \%-73 \%$ and specificity of $76 \%-93 \%[18,20,21,28,29]$. In these studies of PH patients compared to controls, identifying PH based on the PA size, or the correlation between the two, may be artificially affected by the separation in severity of PA pressures between the groups being selected. Additionally, $\mathrm{PH}$ in different clinical contexts may affect the discriminatory ability of the PA measures. For example, the diagnostic accuracy is reduced when looking at patients with ILD (AUC $=0.65)$, with a specificity of $41 \%$ and sensitivity of $86 \%$ for a PA diameter cut-off of $25 \mathrm{~mm}$ [12].

There are a limited number of investigations on how much the measure of the PA size, when used in conjunction with other clinical tools such as the echocardiogram, will add to the diagnosis or exclusion of $\mathrm{PH}$ [14]. However, one interesting study used a regression model to adjust for other variables on $\mathrm{CT}$ in combination with other anthropomorphic variables. In this heterogeneous cohort of 101 hospitalized patients with cardiopulmonary diseases who had a RHC and chest CT performed a mean of three days apart, the main PA and the PA/Ao ratio were poorly predictive of PH. However, the accuracy of the model when adjusted for age, gender, BSA, thoracic diameter, ascending aortic diameter, and pulmonary wedge pressure, improved with an AUC of 0.93 (sensitivity $77 \%$, specificity 89\%) [9] confirming that multiple factors are at play in the relationship between the PA size and PH. 
Our own internal investigations to see if CT measurements of the PA size might help in the diagnostic algorithm for patients with suspected $\mathrm{PH}$ was similar to what others have thus far reported. We retrospectively reviewed 109 patients with suspected PH who underwent both a chest CT and RHC within 180 days apart. The diagnostic categories were; Group $143 \%$, followed by Group $322 \%$, Group 2 18\%, Group 4 9\%, and Group 5 5\%. The main PA diameter normalized to the aortic diameter (PA/Ao) did significantly correlate with the MPAP as measured on a RHC, but weakly $(\mathrm{r}=0.28$, $\mathrm{p}=0.0032$ ). Furthermore, its discriminatory ability for PH was poor with an AUC of 0.61 [44], making its clinical utility in our population unlikely.

\section{Clinical Utility}

It is possible that despite its overall variable diagnostic utility for $\mathrm{PH}$, that the measurements of the PA may have other important clinical implications. However, such relationships appear to be specific to the disease being considered. For example, in a cohort of 3464 patients with Global Initiative for Chronic Obstructive Lung Disease (GOLD) stage II to IV chronic obstructive pulmonary disease (COPD), PA/Ao ratio greater than 1 was associated with subsequent or any COPD exacerbation, but after adjustment for age, presence of gastroesophageal reflux disease, FEV1, the St. George's Respiratory Questionnaire, and prior exacerbations (odds ratio 3.44; 95\% CI 2.78-4.25, p < 0.001) [45]. Although the PA enlargement in this setting could be due to several pathologic mechanisms (e.g., volume fluctuations, hypoxic stress), its ability to predict future exacerbations indicates the potential for PA measures to be clinically useful independent of its ability to diagnose or correlate with any PH.

The association with exacerbations might also hold true for patients with ILD. Matsushita et al. observed that patients who had an acute exacerbation of their ILD had a greater increase in their PA diameter (by $3.15 \mathrm{~mm}$, SD 0.54 vs. $2.89 \mathrm{~mm}$, SD 0.6, p < 0.0001) from a baseline CT, and had a higher $\mathrm{PA} /$ Ao ratio (0.94, SD 0.19 vs. 0.85, SD 0.18, p < 0.0001) [46]. Alterations in hemodynamics and hypoxic vasoconstriction affecting the PA size may be more secondary rather than causal, and whether this might have a clinical role is yet to be determined [46].

In contrast, Boerrigter et al. [47] observed that progressive PA dilatation by MRI during follow-up (942 days, range 242-2,359 days) did not reflect hemodynamic changes in MPAP or cardiac output by RHC in 51 patients with Group $1 \mathrm{PAH}$. The authors suggested that structural changes in elastin and collagen under the influence of an increased pressure might become a cause of PA dilatation independent of hemodynamics. The results suggest that serial PA dimensions are not useful in clinical practice to evaluate the course of the disease, therapeutic response, or change in MPAP.

Finally, in 264 patients with inoperable CTEPH, the PA size was associated with unexpected death [48], even though the causes of death were heterogeneous [45]. Overall, these studies suggest that perhaps the PA dimensions might have other clinical relevance aside from its ability to predict $\mathrm{PH}$, particularly in acute settings. Further study in its role in acute cardiopulmonary decompensations might be of further interest. 


\section{Summary}

In summary, although CT measurements of PA dimensions have shown a correlation with the presence of $\mathrm{PH}$ and $\mathrm{PH}$ severity, the strength of the correlation is highly variable and inadequate for its routine application in clinical practice. Significant variability exists within populations, particularly in the heterogeneous set of diseases that can cause PH. Additional heterogeneity comes from the complex set of physiologic and anatomic factors that disconnect the PA size from PA pressures. Future studies could focus on specific disease subsets and changes in the PA size as a marker of prognosis, disease activity, and treatment response, rather than as an isolated measure. CT measures of PA size are simple and can suggest a possible reason for dyspnea but it should be interpreted cautiously and not be used solely in either screening or guiding management in the patients with suspected $\mathrm{PH}$.

\section{Author Contributions}

KU contributed to conception and design of the study, collection, analysis, and interpretation of the data, drafting and critical revision of the article, and collection/generation of the figures. JPW, TAL, and IDC contributed to the experiments, collection of data, and drafting of the article. CDB contributed to the conception and design of the study and critical revision of the article. ASL contributed to the conception and design of the study, analysis and interpretation of the data, and critical revision of the article. All authors gave final approval of the article.

\section{Conflicts of Interest}

The authors declare no conflict of interest.

\section{References}

1. D'Alonzo, G.E.; Barst, R.J.; Ayres, S.M.; Bergofsky, E.H.; Brundage, B.H.; Detre, K.M.; Fishman, A.P.; Goldring, R.M.; Groves, B.M.; Kernis, J.T.; et al. Survival in patients with primary pulmonary hypertension. Results from a national prospective registry. Ann. Intern. Med. 1991, 115, 343-349.

2. Benza, R.L.; Miller, D.P.; Barst, R.J.; Badesch, D.B.; Frost, A.E.; McGoon, M.D. An evaluation of long-term survival from time of diagnosis in pulmonary arterial hypertension from the REVEAL Registry. Chest 2012, 142, 448-456.

3. Galie, N.; Corris, P.A.; Frost, A.; Girgis, R.E.; Granton, J.; Jing, Z.C.; Klepetko, W.; McGoon, M.D.; McLaughlin, V.V.; Preston, I.R.; et al. Updated treatment algorithm of pulmonary arterial hypertension. J. Am. Coll. Cardiol. 2013, 62, D60-D72.

4. McLaughlin, V.V.; Archer, S.L.; Badesch, D.B.; Barst, R.J.; Farber, H.W.; Lindner, J.R.; Mathier, M.A.; McGoon, M.D.; Park, M.H.; Rosenson, R.S.; et al. ACCF/AHA 2009 expert consensus document on pulmonary hypertension a report of the American College of Cardiology Foundation Task Force on Expert Consensus Documents and the American Heart Association developed in collaboration with the American College of Chest Physicians; American Thoracic Society, Inc.; and the Pulmonary Hypertension Association. J. Am. Coll. Cardiol. 2009, 53, 1573-1619. 
5. Galie, N.; Hoeper, M.M.; Humbert, M.; Torbicki, A.; Vachiery, J.L.; Barbera, J.A.; Beghetti, M.; Corris, P.; Gaine, S.; Gibbs, J.S.; et al. Guidelines for the diagnosis and treatment of pulmonary hypertension: The Task Force for the Diagnosis and Treatment of Pulmonary Hypertension of the European Society of Cardiology (ESC) and the European Respiratory Society (ERS), endorsed by the International Society of Heart and Lung Transplantation (ISHLT). Eur. Heart J. 2009, 30, 2493-2537.

6. Epler, G.R.; McLoud, T.C.; Gaensler, E.A.; Mikus, J.P.; Carrington, C.B. Normal chest roentgenograms in chronic diffuse infiltrative lung disease. N. Engl. J. Med. 1978, 298, 934-939.

7. Zompatori, M.; Bna, C.; Poletti, V.; Spaggiari, E.; Ormitti, F.; Calabro, E.; Tognini, G.; Sverzellati, N. Diagnostic imaging of diffuse infiltrative disease of the lung. Respiration 2004, 71, 4-19.

8. McGoon, M.; Gutterman, D.; Steen, V.; Barst, R.; McCrory, D.C.; Fortin, T.A.; Loyd, J.E. Screening, early detection, and diagnosis of pulmonary arterial hypertension: ACCP evidencebased clinical practice guidelines. Chest 2004, 126, 14S-34S.

9. Chan, A.L.; Juarez, M.M.; Shelton, D.K.; MacDonald, T.; Li, C.S.; Lin, T.C.; Albertson, T.E. Novel computed tomographic chest metrics to detect pulmonary hypertension. BMC Med. Imag. 2011, 11, 7.

10. Burger, I.A.; Husmann, L.; Herzog, B.A.; Buechel, R.R.; Pazhenkottil, A.P.; Ghadri, J.R.; Nkoulou, R.N.; Jenni, R.; Russi, E.W.; Kaufmann, P.A. Main pulmonary artery diameter from attenuation correction CT scans in cardiac SPECT accurately predicts pulmonary hypertension. J. Nucl. Cardiol. 2011, 18, 634-641.

11. Abel, E.; Jankowski, A.; Pison, C.; Luc Bosson, J.; Bouvaist, H.; Ferretti, G.R. Pulmonary artery and right ventricle assessment in pulmonary hypertension: Correlation between functional parameters of ECG-gated CT and right-side heart catheterization. Acta Radiol. 2012, 53, 720-727.

12. Alhamad, E.H.; Al-Boukai, A.A.; Al-Kassimi, F.A.; Alfaleh, H.F.; Alshamiri, M.Q.; Alzeer, A.H.; Al-Otair, H.A.; Ibrahim, G.F.; Shaik, S.A. Prediction of pulmonary hypertension in patients with or without interstitial lung disease: Reliability of CT findings. Radiology 2011, 260, 875-883.

13. Corson, N.; Armato, S.G., 3rd; Labby, Z.E.; Straus, C.; Starkey, A.; Gomberg-Maitland, M. CT-based pulmonary artery measurements for the assessment of pulmonary hypertension. Acad. Radiol. 2014, 21, 523-530.

14. Devaraj, A.; Wells, A.U.; Meister, M.G.; Corte, T.J.; Wort, S.J.; Hansell, D.M. Detection of pulmonary hypertension with multidetector CT and echocardiography alone and in combination. Radiology 2010, 254, 609-616.

15. Edwards, P.D.; Bull, R.K.; Coulden, R. CT measurement of main pulmonary artery diameter. Br. J. Radiol. 1998, 71, 1018-1020.

16. Kam, J.C.; Pi, J.; Doraiswamy, V.; Elnahar, Y.; Abdul-Jawad, S.; DeBari, V.A.; Klukowicz, A.J.; Shamoon, F.; Miller, R.A. CT scanning in the evaluation of pulmonary hypertension. Lung 2013, 191, 321-326.

17. Kuriyama, K.; Gamsu, G.; Stern, R.G.; Cann, C.E.; Herfkens, R.J.; Brundage, B.H. CT-determined pulmonary artery diameters in predicting pulmonary hypertension. Invest. Radiol. 1984, 19, 16-22. 
18. Mahammedi, A.; Oshmyansky, A.; Hassoun, P.M.; Thiemann, D.R.; Siegelman, S.S. Pulmonary artery measurements in pulmonary hypertension: The role of computed tomography. J. Thorac. Imag. 2013, 28, 96-103.

19. Moore, N.R.; Scott, J.P.; Flower, C.D.; Higenbottam, T.W. The relationship between pulmonary artery pressure and pulmonary artery diameter in pulmonary hypertension. Clin. Radiol. 1988, 39, 486-489.

20. Ng, C.S.; Wells, A.U.; Padley, S.P. A CT sign of chronic pulmonary arterial hypertension: the ratio of main pulmonary artery to aortic diameter. J. Thorac. Imag. 1999, 14, 270-278.

21. Sanal, S.; Aronow, W.S.; Ravipati, G.; Maguire, G.P.; Belkin, R.N.; Lehrman, S.G. Prediction of moderate or severe pulmonary hypertension by main pulmonary artery diameter and main pulmonary artery diameter/ascending aorta diameter in pulmonary embolism. Cardiol. Rev. 2006, $14,213-214$.

22. Schmidt, H.C.; Kauczor, H.U.; Schild, H.H.; Renner, C.; Kirchhoff, E.; Lang, P.; Iversen, S.; Thelen, M. Pulmonary hypertension in patients with chronic pulmonary thromboembolism: chest radiograph and CT evaluation before and after surgery. Eur. Radiol. 1996, 6, 817-825.

23. Tan, R.T.; Kuzo, R.; Goodman, L.R.; Siegel, R.; Haasler, G.B.; Presberg, K.W. Utility of CT scan evaluation for predicting pulmonary hypertension in patients with parenchymal lung disease. Medical College of Wisconsin Lung Transplant Group. Chest 1998, 113, 1250-1256.

24. Zisman, D.A.; Karlamangla, A.S.; Ross, D.J.; Keane, M.P.; Belperio, J.A.; Saggar, R.; Lynch, J.P., 3rd; Ardehali, A.; Goldin, J. High-resolution chest CT findings do not predict the presence of pulmonary hypertension in advanced idiopathic pulmonary fibrosis. Chest 2007, 132, 773-779.

25. Haimovici, J.B.; Trotman-Dickenson, B.; Halpern, E.F.; Dec, G.W.; Ginns, L.C.; Shepard, J.A.; McLoud, T.C. Relationship between pulmonary artery diameter at computed tomography and pulmonary artery pressures at right-sided heart catheterization. Massachusetts General Hospital Lung Transplantation Program. Acad. Radiol. 1997, 4, 327-334.

26. Grubstein, A.; Benjaminov, O.; Dayan, D.B.; Shitrit, D.; Cohen, M.; Kramer, M.R. Computed tomography angiography in pulmonary hypertension. Israel Med. Assoc. J. 2008, 10, 117-120.

27. Devaraj, A.; Wells, A.U.; Meister, M.G.; Corte, T.J.; Hansell, D.M. The effect of diffuse pulmonary fibrosis on the reliability of CT signs of pulmonary hypertension. Radiology 2008, 249, 1042-1049.

28. Dornia, C.; Lange, T.J.; Behrens, G.; Stiefel, J.; Muller-Wille, R.; Poschenrieder, F.; Pfeifer, M.; Leitzmann, M.; Manos, D.; Babar, J.L.; et al. Multidetector computed tomography for detection and characterization of pulmonary hypertension in consideration of WHO classification. J. Comput. Assist. Tomo. 2012, 36, 175-180.

29. Iyer, A.S.; Wells, J.M.; Vishin, S.; Bhatt, S.P.; Wille, K.M.; Dransfield, M.T. CT scan-measured pulmonary artery to aorta ratio and echocardiography for detecting pulmonary hypertension in severe COPD. Chest 2014, 145, 824-832.

30. Rajaram, S.; Swift, A.J.; Capener, D.; Elliot, C.A.; Condliffe, R.; Davies, C.; Hill, C.; Hurdman, J.; Kidling, R.; Akil, M.; et al. Comparison of the diagnostic utility of cardiac magnetic resonance imaging, computed tomography, and echocardiography in assessment of suspected pulmonary arterial hypertension in patients with connective tissue disease. J. Rheumatol. 2012, 39, 1265-1274. 
31. Lange, T.J.; Dornia, C.; Stiefel, J.; Stroszczynski, C.; Arzt, M.; Pfeifer, M.; Hamer, O.W. Increased pulmonary artery diameter on chest computed tomography can predict borderline pulmonary hypertension. Pulmonary Circulation 2013, 3, 363-368.

32. McCall, R.K.; Ravenel, J.G.; Nietert, P.J.; Granath, A.; Silver, R.M. Relationship of main pulmonary artery diameter to pulmonary arterial pressure in scleroderma patients with and without interstitial fibrosis. J. Comput. Assist. Tomo. 2014, 38, 163-168.

33. Truong, Q.A.; Massaro, J.M.; Rogers, I.S.; Mahabadi, A.A.; Kriegel, M.F.; Fox, C.S.; O'Donnell, C.J.; Hoffmann, U. Reference values for normal pulmonary artery dimensions by noncontrast cardiac computed tomography: The Framingham Heart Study. Circulation 2012, 5, 147-154.

34. Edwards, W.D.; Edwards, J.E. Clinical primary pulmonary hypertension: Three pathologic types. Circulation 1977, 56, 884-888.

35. Heath, D.; Smith, P.; Gosney, J.; Mulcahy, D.; Fox, K.; Yacoub, M.; Harris, P. The pathology of the early and late stages of primary pulmonary hypertension. Br. Heart J. 1987, 58, 204-213.

36. Simonneau, G.; Gatzoulis, M.A.; Adatia, I.; Celermajer, D.; Denton, C.; Ghofrani, A.; Gomez Sanchez, M.A.; Krishna Kumar, R.; Landzberg, M.; Machado, R.F.; et al. Updated clinical classification of pulmonary hypertension. J. Am. Coll. Cardiol. 2013, 62, D34-D41.

37. Mitsumori, L.M.; Wang, E.; May, J.M.; Lockhart, D.W.; Branch, K.R.; Dubinsky, T.J.; Shuman, W.P. Triphasic contrast bolus for whole-chest ECG-gated 64-MDCT of patients with nonspecific chest pain: Evaluation of arterial enhancement and streak artifact. Am. J. Roentgenol. 2010, 194, W263-W271.

38. Weininger, M.; Barraza, J.M.; Kemper, C.A.; Kalafut, J.F.; Costello, P.; Schoepf, U.J. Cardiothoracic CT angiography: current contrast medium delivery strategies. Am. J. Roentgenol. 2011, 196, W260-W272.

39. Mortimer, A.M.; Singh, R.K.; Hughes, J.; Greenwood, R.; Hamilton, M.C. Use of expiratory CT pulmonary angiography to reduce inspiration and breath-hold associated artefact: contrast dynamics and implications for scan protocol. Clin. Radiol. 2011, 66, 1159-1166.

40. Karazincir, S.; Balci, A.; Seyfeli, E.; Akoglu, S.; Babayigit, C.; Akgul, F.; Yalcin, F.; Egilmez, E. $\mathrm{CT}$ assessment of main pulmonary artery diameter. Diagn. Interv. Radiol. 2008, 14, 72-74.

41. Kovacs, G.; Berghold, A.; Scheidl, S.; Olschewski, H. Pulmonary arterial pressure during rest and exercise in healthy subjects: A systematic review. Eur. Resp. J. 2009, 34, 888-894.

42. Nevsky, G.; Jacobs, J.E.; Lim, R.P.; Donnino, R.; Babb, J.S.; Srichai, M.B. Sex-specific normalized reference values of heart and great vessel dimensions in cardiac CT angiography. Am. J. Roentgenol. 2011, 196, 788-794.

43. Condliffe, R.; Radon, M.; Hurdman, J.; Davies, C.; Hill, C.; Akil, M.; Guarasci, F.; Rajaram, S.; Swift, A.J.; Wragg, Z.; et al. CT pulmonary angiography combined with echocardiography in suspected systemic sclerosis-associated pulmonary arterial hypertension. Rheumatology 2011, 50, 1480-1486.

44. Darin Carabenciov, T.L.; Burger, C.; Lee, A. Correlation Between CT Measured Pulmonary Artery Size And Invasively Confirmed Pulmonary Artery Pressures. Am. J. Resp. Crit. Care Med. 2012, A1886. 
45. Wells, J.M.; Washko, G.R.; Han, M.K.; Abbas, N.; Nath, H.; Mamary, A.J.; Regan, E.; Bailey, W.C.; Martinez, F.J.; Westfall, E.; et al. Pulmonary arterial enlargement and acute exacerbations of COPD. N. Engl. J. Med. 2012, 367, 913-921.

46. Matsushita, S.; Matsuoka, S.; Yamashiro, T.; Fujikawa, A.; Yagihashi, K.; Kurihara, Y.; Nakajima, Y. Pulmonary arterial enlargement in patients with acute exacerbation of interstitial pneumonia. Clin. Imag. 2014, 38, 454-457.

47. Boerrigter, B.; Mauritz, G.J.; Marcus, J.T.; Helderman, F.; Postmus, P.E.; Westerhof, N.; VonkNoordegraaf, A. Progressive dilatation of the main pulmonary artery is a characteristic of pulmonary arterial hypertension and is not related to changes in pressure. Chest 2010, 138, 1395-1401.

48. Zylkowska, J.; Kurzyna, M.; Florczyk, M.; Burakowska, B.; Grzegorczyk, F.; Burakowski, J.; Wieteska, M.; Oniszh, K.; Biederman, A.; Wawrzynska, L.; et al. Pulmonary artery dilatation correlates with the risk of unexpected death in chronic arterial or thromboembolic pulmonary hypertension. Chest 2012, 142, 1406-1416.

(C) 2014 by the authors; licensee MDPI, Basel, Switzerland. This article is an open access article distributed under the terms and conditions of the Creative Commons Attribution license (http://creativecommons.org/licenses/by/3.0/). 